\title{
On the Borchers Class of a Non-Commutative Field
}

\author{
Daniel H.T. Franco*† \\ Universidade Federal do Espírito Santo (UFES) \\ Departamento de Física - Campus Universitário de Goiabeiras \\ CEP:29060-900 - Vitória - ES - Brasil \\ November, 2018
}

\begin{abstract}
In this paper, we arrive at the notion of equivalence classes of a non-commutative field exploring some ideas by Soloviev to nonlocal quantum fields. Specifically, an equivalence relation between non-commutative fields is formulated by replacing the weak relative locality condition by a weak relative asymptotic commutativity property, generalizing the notion of relative locality proved by Borchers in the framework of local QFT. We restrict ourselves to the simplest case of a scalar field theory with space-space non-commutativity.
\end{abstract}

Keywords: Non-commutative theory, axiomatic field theory.

\section{Introduction}

At the present, a considerable effort has been made to clarify the structural aspects of non-commutative field theories (NCFT) from an axiomatic standpoint. The first paper within this context is due to Álvarez-Gaumé and Vásquez-Mozo [1]. By modifying the standard Wightman axioms, using as guiding principles the breaking of Lorentz symmetry down to the subgroup $O(1,1) \times S O(2)$, which leaves invariant the commutation relations for the coordinate operators $\left[\widehat{x}_{\mu}, \widehat{x}_{\nu}\right]=i \theta_{\mu \nu}$, and the relaxation of local commutativity to make it compatible with the causal structure of the theory, described by the light-wedge associated with the $O(1,1)$ factor of the kinematical symmetry group, they have demonstrated the validity of the CPT theorem for NCFT with space-space non-commutativity. More recently, Chaichian et al. [2] have proposed new Wightman functions as vacuum expectation values of products of field operators in the non-commutative space-time. These Wightman functions involve the $\star$-product. In the case of only space-space noncommutativity $\left(\theta_{0 i}=0\right)$, they have proved the CPT and Spin-Statistics theorem, for

\footnotetext{
* On leave from the Centro de Estudos de Física Teórica, Belo Horizonte, MG, Brasil.

†e-mail: dhtf@terra.com.br
} 
the simplest case of a scalar field, using the non-commutative form of the Wightman functions.

As it was emphasized in [1], a source of difficulties in formulating NCFT which satisfy the adapted axioms has been the very harmful UV/IR mixing, which is probably the most surprising feature of these theories. The existence of hard infrared singularities in the nonplanar sector of the theory, induced by uncancelled quadratic ultraviolet divergences, can result in two kinds of problems: they can destroy the tempered nature of the Wightman functions and/or they can introduce tachyonic states in the spectrum, so the modified postulate of local commutativity is not preserved. In order to find a way of avoiding these problems, and due to the highly nonlocal character of the commutation relations $\left[\widehat{x}_{\mu}, \widehat{x}_{\nu}\right]=i \theta_{\mu \nu}$, one might want to consider other spaces of distributions. Having this in mind, in [3], we adopted the space of distributions which has been explored some time by Lücke [4]-[6] and Soloviev [7]-[10]. These authors have shown that one adequate solution to treat field theories with nonlocal interactions, it is to take the fields to be averaged with test functions belonging to the space $\mathcal{S}^{0}\left(\mathbb{R}^{n}\right)$, consisting of the restrictions to $\mathbb{R}^{n}$ of entire analytic functions on $\mathbb{C}^{n}$, whose Fourier transform is just the Schwartz space $\mathscr{D}\left(\mathbb{R}^{n}\right)$ of $C^{\infty}$ functions of compact support. The space $\mathcal{S}^{0}\left(\mathbb{R}^{n}\right)$ is the smallest space among the Gelfand-Shilov spaces [11], $\mathcal{S}^{\beta}\left(\mathbb{R}^{n}\right)$, where $0 \leq \beta<1$, which naturally allows us to treat a theory of nonlocalizable fields. Elements in the dual space $\mathcal{S}^{\prime 0}$ of the space of entire functions are called analytic functionals. Because the elements in $\mathcal{S}^{0}$ are entire functions, the locality axiom cannot be formulated in the usual way, i.e., there is no sensible notion of support for distributions in $\mathcal{S}^{\prime 0}$. Nevertheless, Soloviev has shown that the functionals of this class retain a kind of angular localizability, which ensures the existence of minimal carrier cones of the distributions in $\mathcal{S}^{\prime 0}$, which allows us handling the analytic functionals of class $\mathcal{S}^{\prime 0}$, in most cases, as easily as tempered distributions. This replaces the notion of support for nonlocalizable distributions and leads to a natural generalization of the local commutativity. With this, we have been able of reaching a conclusion about the validity of the CPT and Spin-Statistics theorems in NCFT, in the case of only space-space noncommutativity [3].

In this paper, following our previous paper [3], we define what is meant by an equivalence class of quantum fields for a non-commutative field directly applying some ideas by Soloviev to nonlocal quantum fields in [10]. In ordinary field theories this was first described by Borchers $[12,13]$. We restrict ourselves to the simplest case of a scalar field theory with space-space non-commutativity.

The article is organized as follows. In Section 2, the necessary modifications of the Wightman axioms to include the case of NCFT are described. In Section 3, we outline the arguments that lead to a natural extension of the Borchers classes of quantum fields. Section 4 contains our final considerations. An informal synopsis of some results by Soloviev about carrier cones of analytic functionals is given in an Appendix. 


\section{Modifying of the Wightman Axioms for a NCFT}

We shall assume a NCFT with the field operators $\varphi\left(f_{1}\right), \ldots, \varphi\left(f_{n}\right)$ fulfilling all the Wightman axioms except by following modifications: $(a)$ fields are operator-valued generalized functions living in an appropriate space of functions $f(x) \in \mathcal{S}^{0}\left(\mathbb{R}^{4 n}\right)$, the space of entire analytic test functions, $(b)$ the local commutativity is replaced by the asymptotic variant in the sense of Soloviev, which can be understood saying that two operators $\varphi(f)$ and $\varphi(g)$, at two distinct points $x_{1}$ and $x_{2}$, can only be distinguished if the relative spatial distance between the two points $x_{1}$ and $x_{2}$ is sufficiently large.

Fields are Analytical Functionals. The fields are operator-valued generalized functions on $\mathcal{S}^{0}\left(\mathbb{R}^{4 n}\right)$, the space of entire analytic test functions. In particular, we will consider only one neutral scalar field $\varphi(x)$. We denote by $D_{0}$ the minimal common invariant domain, which is assumed to be dense, of the field operators in the Hilbert space $\mathscr{H}$ of states, i.e., the vector subspace of $\mathscr{H}$ that is spanned by the vacuum state $\left|\Omega_{o}\right\rangle$ and by various vectors of the form

$$
\varphi\left(f_{1}\right) \star \cdots \star \varphi\left(f_{n}\right)\left|\Omega_{0}\right\rangle=e^{\frac{i}{2} \theta^{\mu \nu} \sum_{i<j} \frac{\partial}{\partial x_{i}^{\mu}} \frac{\partial}{\partial x_{j}^{\nu}}} \varphi\left(f_{1}\right) \cdots \varphi\left(f_{n}\right)\left|\Omega_{o}\right\rangle
$$

where $f_{i}\left(x_{i}\right) \in \mathcal{S}^{0}\left(\mathbb{R}^{4}\right)$, i.e., we axiomatize NCFT in the sense of a field theory on a non-commutative space-time encoded by a Moyal product on the test function algebra. It should be noted that, the space $\mathcal{S}^{0}$, being Fourier-isomorphic to $\mathscr{D}$, is nuclear. Therefore, the $n$-point vacuum expectation values uniquely determine Wightman generalized functions $\mathscr{W}_{\star} \in \mathcal{S}^{\prime 0}\left(\mathbb{R}^{4 n}\right)$ :

$$
\mathscr{W}_{\star}\left(f_{1} \otimes \cdots \otimes f_{n}\right) \stackrel{\text { def }}{=}\left\langle\Omega_{o}\left|\varphi\left(f_{1}\right) \star \cdots \star \varphi\left(f_{n}\right)\right| \Omega_{o}\right\rangle .
$$

Here $\left(f_{1} \otimes \cdots \otimes f_{n}\right)\left(x_{1}, \ldots, x_{n}\right)=f_{1}\left(x_{1}\right) \cdots f_{n}\left(x_{n}\right)$ is considered as an element of $\mathcal{S}^{0}\left(\mathbb{R}^{4 n}\right)$, where $\mathbb{R}^{4 n}$ is the space of $n$-tuple of Minkowski vectors $\left(x_{1}, \ldots, x_{n}\right)$. Eq.(2.1) represents the Weyl form of the operator-valued Wightman functions $\mathscr{W}_{n}\left(f_{1} \otimes \cdots \otimes f_{n}\right)$ proposed recently by Chaichian et al. [2] (apparently, a similar definition of Wightman functions for the non-commutative case was used in [16]). The relation between $\mathscr{W}_{\star}\left(f_{1} \otimes \cdots \otimes f_{n}\right)$ defined above and the ordinary Wightman functions $\mathscr{W}_{n}\left(f_{1} \otimes \cdots \otimes f_{n}\right)$ is given by

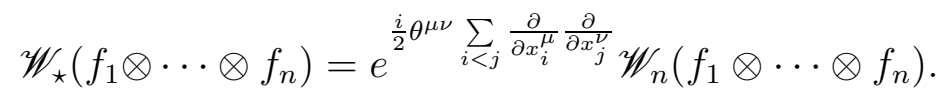

This is a consequence of the product

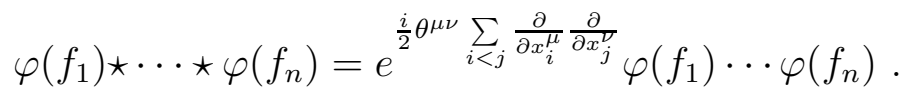

For coinciding points $x_{1}=x_{2}=\cdots=x_{n}$ the product (2.2) becomes identical to the multiple Moyal $\star$-product. The use of the new Wightman functions (2.1) suggests that the corresponding QFT is genuinely a NCFT [2]. 
Asymptotic Commutativity. If $f$ and $g$ are two test functions in $\mathcal{S}^{0}\left(\mathbb{R}^{4}\right)$, then the fields $\varphi(f)$ and $\varphi(g)$ are said to commute asymptotically for sufficiently large space-like separation of their arguments, if the functional

$$
\begin{aligned}
f & =\left\langle\Theta,[\varphi(f), \varphi(g)]_{\star} \Psi\right\rangle \\
& =\langle\Theta,(\varphi(f) \star \varphi(g)-\varphi(g) \star \varphi(f)) \Psi\rangle,
\end{aligned}
$$

is carried by the closed cone $\bar{V}_{e}^{2} \times \mathbb{R}^{4}$, for any vectors $\Theta, \Psi \in D_{0}$, where $\bar{V}_{e}^{2}=\left\{\left(x_{e}, x_{e}^{\prime}\right) \in\right.$ $\left.\mathbb{R}^{4} \mid\left(x_{e}-x_{e}^{\prime}\right)^{2} \geq 0\right\}$. Here, following the Ref. [1], we call the coordinates $x_{e}=\left(x^{0}, x^{1}\right)$ "electrical" coordinates and $\vec{x}_{m}=\left(x^{2}, x^{3}\right)$ "magnetic" coordinates.

In [1] the condition that the functional (2.3) is carried by the closed cone $\bar{V}_{e}^{2} \times \mathbb{R}^{4}$ has been supported by the $S O(1,1) \times S O(2)$ symmetry, which is the feature arising when one has only spatial non-commutativity. This fact leads the authors of [1] to argue that the notion of a light cone is generally modified to that of a light wedge. More recently, Chu et al. [17] have shown that the reduction from light cone to light wedge is a generic effect for non-commutative geometry and it is independent of the type of Lorentz symmetry breaking interaction.

Remark 1 . The analysis based in the symmetry $S O(1,1) \times S O(2)$ shows an inconvenience: particles cannot be classified according to the 4-dimensional Wigner particle concept. However, the recent work of Chaichian et al [18] shows that this can be by passed invoking the concept of twisted Poincaré symmetry. From now on, we will assume that our fields are transforming according to representations of the twisted Poincaré group. This implies that the Wightman functionals defined in (2.1) satisfy the twisted Poincaré covariance condition.

In our arguments the equality $\mathcal{S}^{0}\left(\mathbb{R}^{4} \times \mathbb{R}^{4}\right)=\mathcal{S}^{0}\left(\mathbb{R}^{4}\right) \widehat{\otimes}_{i} \mathcal{S}^{0}\left(\mathbb{R}^{4}\right)$ plays an important role, where the index $i$ indicates that the tensor product is endowed with the inductive topology and the hat means the corresponding completion. By definition of the inductive topology, the dual space of $\mathcal{S}^{0}\left(\mathbb{R}^{4}\right) \widehat{\otimes}_{i} \mathcal{S}^{0}\left(\mathbb{R}^{4}\right)$ is isomorphic to the space of separately continuous functionals on $\mathcal{S}^{0}\left(\mathbb{R}^{4}\right) \times \mathcal{S}^{0}\left(\mathbb{R}^{4}\right)$ (see $\left.[9,10]\right)$. By result $\mathbf{R} .2$ in Appendix, the space $\mathcal{S}^{0}\left(\mathbb{R}^{4}\right)$ is dense in $\mathcal{S}^{0}(U)$, where $U$ is any open cone in $\mathbb{R}^{4}$ such that $\bar{V}_{e}^{2} \backslash\{0\} \subset U$. Hence, the functional $f$ is carried by the closed cone $\bar{V}_{e}^{2} \times \mathbb{R}^{4}$. In addition, a consideration analogous to that of Lemma 3 in [9] shows that if we introduce, for $0 \leq j \leq n$ and $n=0,1,2, \ldots$, Wightman functions $\mathscr{W}_{\star} \in \mathcal{S}^{\prime 0}\left(\mathbb{R}^{4(n+2)}\right)$ defined by

$$
\begin{aligned}
\mathscr{W}_{\star}\left(f_{1} \otimes \cdots \otimes f_{j} \otimes f \otimes g \otimes f_{j+1} \otimes \cdots \otimes f_{n}\right) \pm \\
\mathscr{W}_{\star}\left(f_{1} \otimes \cdots \otimes f_{j} \otimes g \otimes f \otimes f_{j+1} \otimes \cdots \otimes f_{n}\right),
\end{aligned}
$$

it follows from the asymptotic commutativity condition that $\mathscr{W}_{\star}$ defined on $\mathcal{S}^{0}\left(\mathbb{R}^{4(n+2)}\right)$ has a continuous extension to the space $\mathcal{S}^{0}\left(\mathbb{R}^{4 j} \times\left(U \times \mathbb{R}^{4}\right) \times \mathbb{R}^{4(n-j)}\right)$, where $U$ is any open cone in $\mathbb{R}^{4}$ such that $\bar{V}_{e}^{2} \backslash\{0\} \subset U$. Then, $\mathscr{W}_{\star}$ is carried by the closed cone $\mathbb{R}^{4 j} \times$ $\left(\bar{V}_{e}^{2} \times \mathbb{R}^{4}\right) \times \mathbb{R}^{4(n-j)}$. 
Remark 2. From the estimates of the behavior of test functions belonging to space $\mathcal{S}^{0}$ over cones (see Eq.(A.1)), the feature of the functional (2.3) to be carried by $\bar{V}_{e}^{2} \times \mathbb{R}^{4}$ is equivalent to the property of this functional to decrease faster than any linear exponential function in the complement of $\bar{V}_{e}^{2} \times \mathbb{R}^{4}$. This statement is a special case of Theorem 2 (Convolution Theorem) in [19]. Particularly, this brings us back to an old work due to Borchers-Pohlmeyer [20], who considered the theory of a tempered scalar field and replaced the microcausality condition by an apparently weaker condition: they established that the bound of the form

$$
\left|\left\langle\Omega_{o}\left|\left[\varphi\left(x_{1}\right), \varphi\left(x_{2}\right)\right] \varphi\left(x_{3}\right) \cdots \varphi\left(x_{n}\right)\right| \Omega_{o}\right\rangle\right| \leq C_{n} e^{-\epsilon\left|\left(x_{1}-x_{2}\right)^{2}\right|^{\alpha / 2}}, \quad \text { for } \quad \alpha>1,
$$

on the behavior of the commutators at those points $\left(x_{1}, x_{2}, \ldots, x_{n}\right)$ which belong to the open cone $\mathscr{J}_{n}$, the Jost cone [21], together with $\left(x_{2}, x_{1}, \ldots, x_{n}\right)$ results in the strict local commutativity. In other words, they proved that a vanishing of the commutator of the fields in the relative spatial distance $\left(x_{1}-x_{2}\right)$ which is faster than a certain exponential decay leads back to a microlocal theory. However, as observed by Soloviev [9], the asymptotic commutativity condition being applied to the tempered field does not amount to the naive bound (2.4) and does not imply local commutativity. The asymptotic commutativity condition indicates a fast decrease not of the commutator itself but of the result of smoothing it by convolution with appropriate test functions [19].

\section{The Notion of Equivalence Classes of a NC Field}

As it is well-known, the Borchers class of a quantum field is a direct consequence of the $\mathrm{CPT}$ theorem. For this reason, we shall recall the validity of this theorem within the context of a NCFT $[2,3]$. For simplicity, we will assume a NCFT given in terms of a single neutral scalar field, in the case of only spatial non-commutativity.

Theorem 3.1 (Modified CPT Theorem). A non-commutative scalar field theory symmetric under the CPT-operation $\Theta$ is equivalent to the weak asymptotic commutative condition.

To prove the Theorem 3.1, we must develop our machinery a little further.

Proposition 3.2. The functional $\mathscr{F}(f)=\mathscr{W}_{\star}\left(f_{1} \otimes \cdots \otimes f_{n}\right)-\mathscr{W}_{\star}\left(\widehat{f}_{1} \otimes \cdots \otimes \widehat{f}_{n}\right)$, where $\widehat{f}_{i}\left(x_{i}\right)=f_{i}\left(-x_{i}\right)$, is carried by the complement of the Jost real points $\mathscr{J}_{n}$, characterized by the set

$$
\left\{\left(x_{1}, \ldots, x_{n}\right) \in \mathbb{R}^{4 n} \mid\left(\sum_{i=1}^{n-1} \lambda_{i}\left(x_{e_{i}}-x_{e_{i+1}}\right)\right)^{2}<0\right\}
$$

for an arbitrary set of $\lambda_{i}$ satisfying the conditions $\lambda_{i} \geq 0$ and $\sum_{i=1}^{n-1} \lambda_{i}>0$. 
Proof. The proof follows using arguments paralleling the analysis of the proof of the Proposition 4.1 in [3], taking into account that the new Wightman functions no affect the analytic continuation of these functions to the complex plane with respect the "electrical" coordinates $x_{e}=\left(x^{0}, x^{1}\right)$.

Remark 3. According to Álvarez-Gaumé and Vásquez-Mozo [1], for $n>2$ the Jost points are formed by $\left(x_{e_{i}}-x_{e_{i+1}}\right)^{2}<0$ with the condition that $x_{i}^{1}-x_{i+1}^{1}>0$.

Definition 3.3. The non-commutative quantum field $\varphi(x)$ defined on the test function space $\mathcal{S}^{0}\left(\mathbb{R}^{4}\right)$ is said to satisfy the weak asymptotic commutativity (WAC) condition if the functional

$$
\mathscr{W}_{\star}\left(f_{1} \otimes \cdots \otimes f_{n}\right)-\mathscr{W}_{\star}\left(f_{n} \otimes \cdots \otimes f_{1}\right),
$$

is carried by the set $\complement \mathscr{J}_{n}$ complementary to the Jost points.

Proof of Theorem 3.1. The CPT invariance condition is derived by requiring that the CPT operator $\Theta$ be antiunitary - see $[22,23,24]$ :

$$
\langle\Theta \Xi \mid \Theta \Psi\rangle=\langle\Psi \mid \Xi\rangle
$$

this means that the CPT operator leaves invariant all transition probabilities of the theory. In the case of a NCFT, the operator $\Theta$ can be constructed in the ordinary way. Taking the vector states as $\langle\Xi|=\left\langle\Omega_{o}\right|$ and $|\Psi\rangle=\varphi\left(f_{n}\right) \star \cdots \star \varphi\left(f_{1}\right)\left|\Omega_{o}\right\rangle$ we shall express both sides of (3.1) in terms of NC Wightman functions. For the left-hand side of (3.1) we can use directly the CPT transformation properties of the field operators, which for a neutral scalar field is equal to $\Theta \varphi(f) \Theta^{-1}=\varphi(\widehat{f})$. Using the CPT-invariance of the vacuum state, $\Theta\left|\Omega_{o}\right\rangle=\left|\Omega_{o}\right\rangle$, the left-hand side of (3.1) becomes:

$$
\begin{aligned}
\langle\Theta \Xi \mid \Theta \Psi\rangle & =\left\langle\Theta \Omega_{o}\right| \Theta\left(\varphi\left(f_{n}\right) \star \cdots \star \varphi\left(f_{1}\right)\left|\Omega_{o}\right\rangle\right. \\
& =\mathscr{W}_{\star}\left(\widehat{f}_{n} \otimes \cdots \otimes \widehat{f}_{1}\right) .
\end{aligned}
$$

In order to express the right-hand side of (3.1), we take the Hermitian conjugates of the vectors $|\Psi\rangle$ and $\langle\Xi|$, to obtain:

$$
\langle\Psi \mid \Xi\rangle=\mathscr{W}_{\star}\left(f_{1} \otimes \cdots \otimes f_{n}\right)
$$

Putting together (3.1) with (3.2) and (3.3), we obtain the CPT invariance condition in terms of NC Wightman functions as

$$
\mathscr{W}_{\star}\left(f_{1} \otimes \cdots \otimes f_{n}\right)=\mathscr{W}_{\star}\left(\widehat{f}_{n} \otimes \cdots \otimes \widehat{f}_{1}\right) .
$$

Now, we subtract the functional $\mathscr{W}_{\star}\left(\widehat{f}_{1} \otimes \cdots \otimes \widehat{f}_{n}\right)$ from the left-hand and right-hand sides of (3.4) and obtain the expression:

$$
\left[\mathscr{W}_{\star}\left(f_{1} \otimes \cdots \otimes f_{n}\right)-\mathscr{W}_{\star}\left(\widehat{f}_{1} \otimes \cdots \otimes \widehat{f}_{n}\right)\right]=\left[\mathscr{W}_{\star}\left(\widehat{f}_{n} \otimes \cdots \otimes \widehat{f}_{1}\right)-\mathscr{W}_{\star}\left(\widehat{f}_{1} \otimes \cdots \otimes \widehat{f}_{n}\right)\right] .
$$


By Proposition 3.2, the difference functional on the left-hand side, denoted by $\mathscr{F}(f)$, is carried by set $\complement \mathscr{J}_{n}$. It follows that the functional on the right-hand side is also carried by this set. This means that the WAC is fulfilled, taking into consideration the symmetry $\mathscr{J}_{n}=-\mathscr{J}_{n}$ of the Jost points. The reverse is also easily proved. If the WAC is satisfied, then the difference $\mathscr{W}_{\star}\left(f_{1} \otimes \cdots \otimes f_{n}\right)-\mathscr{W}_{\star}\left(\widehat{f}_{n} \otimes \cdots \otimes \widehat{f}_{1}\right)$ is carried by $\complement \mathscr{J}_{n} \neq \mathbb{R}^{2 n} \times \mathbb{R}^{2 n}$. On the other hand, by virtue of the spectral condition, the Fourier transform of this difference has support in the properly convex cone

$$
\left\{\left(p_{1}, \ldots, p_{n}\right) \in \mathbb{R}^{4 n} \mid \sum_{j=1}^{n} p_{j}=0, \sum_{j=1}^{k} p_{j} \in \bar{V}_{+}, k=1, \ldots, n-1\right\}
$$

Therefore, the CPT invariance holds identically by Theorem 4 in [8], which asserts that $\mathscr{W}_{\star}\left(f_{1} \otimes \cdots \otimes f_{n}\right)-\mathscr{W}_{\star}\left(\widehat{f}_{n} \otimes \cdots \otimes \widehat{f}_{1}\right) \equiv 0$, since the property of this functional of having its Fourier transform supported by the aforementioned properly convex cone requires that each carrier cone of $\mathscr{W}_{\star}\left(f_{1} \otimes \cdots \otimes f_{n}\right)-\mathscr{W}_{\star}\left(\widehat{f}_{n} \otimes \cdots \otimes \widehat{f}_{1}\right)$ cannot be different from the whole space $\mathbb{R}^{2 n} \times \mathbb{R}^{2 n}$.

Finally, the Borchers class of quantum fields for a NCFT is a consequence of the following theorem.

Theorem 3.4. Suppose $\varphi(f)$ is a field satisfying the assumptions of Theorem 3.1 and $\Theta$ is the corresponding CPT-symmetry operator. Suppose $\psi(f)$ is another field transforming under the same representation of the twisted Poincaré group, with the same domain of definition. Suppose that the functional $\left\langle\Omega_{o}\right| \varphi\left(f_{1}\right) \star \cdots \star \varphi\left(f_{m}\right) \star \psi(f) \star \varphi\left(f_{m+1}\right) \star \cdots \star$ $\varphi\left(f_{n}\right)\left|\Omega_{o}\right\rangle-\left\langle\Omega_{o}\left|\varphi\left(f_{n}\right) \star \cdots \star \varphi\left(f_{m+1}\right) \star \psi(f) \star \varphi\left(f_{m}\right) \star \cdots \star \varphi\left(f_{1}\right)\right| \Omega_{o}\right\rangle$ is carried by $\complement \mathscr{J}_{n+1}$. Then $\Theta$ implements the CPT symmetry for $\psi(f)$ as well and the fields $\varphi, \psi$ satisfy the weak asymptotic commutativity condition.

Proof. Applying the same arguments as above, we find that

$$
\begin{aligned}
&\left\langle\Omega_{o}\left|\varphi\left(f_{1}\right) \star \cdots \star \varphi\left(f_{m}\right) \star \psi(f) \star \varphi\left(f_{m+1}\right) \star \cdots \star \varphi\left(f_{n}\right)\right| \Omega_{o}\right\rangle= \\
&\left\langle\Omega_{o}\left|\varphi\left(\widehat{f}_{n}\right) \star \cdots \star \varphi\left(\widehat{f}_{m+1}\right) \star \psi(\widehat{f}) \star \varphi\left(\widehat{f}_{m}\right) \star \cdots \star \varphi\left(\widehat{f}_{1}\right)\right| \Omega_{o}\right\rangle
\end{aligned}
$$

If $\Theta$ is the CPT operator for the field $\varphi$, it follows that for any $\Psi$

$$
\left\langle\Theta \Xi\left|\Theta \psi(f) \Theta \Theta^{-1}\right| \Psi\right\rangle=\langle\overline{\Xi|\psi(f)| \Psi}\rangle
$$

and if

$$
|\Xi\rangle=\varphi\left(f_{m}\right) \star \cdots \star \varphi\left(f_{1}\right)\left|\Omega_{o}\right\rangle \quad \text { and } \quad|\Psi\rangle=\varphi\left(f_{m+1}\right) \star \cdots \star \varphi\left(f_{n}\right)\left|\Omega_{o}\right\rangle \text {, }
$$

then

$$
|\Theta \Xi\rangle=\varphi\left(\widehat{f}_{m}\right) \star \cdots \star \varphi\left(\widehat{f}_{1}\right)\left|\Omega_{o}\right\rangle \quad \text { and } \quad|\Theta \Psi\rangle=\varphi\left(\widehat{f}_{m+1}\right) \star \cdots \star \varphi\left(\widehat{f}_{n}\right)\left|\Omega_{o}\right\rangle \text {. }
$$


Using (3.5), we obtain

$$
\langle\Psi|\psi(f)| \Xi\rangle=\langle\Theta \Xi|\psi(\widehat{f}) \Theta| \Psi\rangle,
$$

which when compared with (3.6) leads to

$$
\Theta \psi(f) \Theta^{-1}=\psi(\widehat{f}) .
$$

Thus, the operator $\Theta$ transforms $\psi$ correctly, as was to be proved.

Corollary 3.5 (Transitivity of Weak Relative Asymptotic Commutativity). The weak relative asymptotic commutativity property is transitive in the sense that if each of the fields $\psi_{1}, \psi_{2}$ satisfies the assumptions of Theorem 3.4, then there is a CPT-symmetry operator common to the fields $\left\{\varphi, \psi_{1}, \psi_{2}\right\}$ and by Theorem 3.1, the weak relative asymptotic commutativity condition is satisfied not only for $\left\{\psi_{1}, \psi_{2}\right\}$ but also for $\left\{\varphi, \psi_{1}, \psi_{2}\right\}$.

\section{Final Considerations}

In this work, we continue applying some ideas by Soloviev [7]-[10] as an alternative description towards an axiomatic formulation of non-commutative quantum field theory. Following our previous paper [3], we have extended the Borchers class of quantum fields for a NCFT, by replacing the weak relative locality condition by a weak relative asymptotic commutativity property. We restrict ourselves to the simplest case of a neutral scalar field, in the case of spatial non-commutativity. An important consequence of this result is that the Theorem 4.20 in [22] on S-equivalence of quantum fields can be generalized for NCFT, by adapting the Theorem 6 in [10] to the basic postulates of Section 2 .

\section{Acknowledgments}

I would like to thank Professor O. Piguet for his kind invitations at the Departamento de Física, Universidade Federal do Espírito Santo (UFES) and C.P. Constantinidis for encouragement.

\section{A Angular Localizability}

In this appendix, for sake of completeness, we recall some results by Soloviev which make possible to extend the basic results of axiomatic approach $[22,23,24]$ to nonlocal interactions - the reader is referred to [7]-[10] and references therein for details.

We start recalling that the space of test functions composed by entire analytic functions is such that the following estimate holds:

$$
|f(z)| \leq C_{N}(1+|x|)^{-N} e^{b|y|}
$$

where $z=x+i y$ and $N \in \mathbb{N}$. $C_{N}$ and $b$ are positive constants depending on $f$. The space of functions satisfying the estimate above, with fixed $b$, is denoted as $\mathcal{S}^{0, b}$, while in 
nonlocal field theory the union $\cup_{b>0} \mathcal{S}^{0, b}$ is denoted as $\mathcal{S}^{0}$. Together with space $\mathcal{S}^{0}\left(\mathbb{R}^{n}\right)$, we introduce a space associated with closed cones $K \subset \mathbb{R}^{n}$. One recalls that $K$ is called a cone if $x \in K$ implies $\lambda x \in K$ for all $\lambda>0$. Let $U$ be an open cone in $\mathbb{R}^{n}$. For each $U$, one assigns a space $\mathcal{S}^{0}(U)$ consisting of those entire analytic functions on $\mathbb{C}^{n}$, that satisfy the inequalities

$$
|f(z)| \leq C_{N}(1+|x|)^{-N} e^{b|y|+b d(x, U)}
$$

with $d(x, U)$ being the distance from the point $x$ to the cone $U$. Here, the norm in $\mathbb{R}^{n}$ is assumed to be Euclidean. This space can naturally be given a topology by regarding it as the inductive limit of the family of countably normed spaces $\mathcal{S}^{0, b}(U)$ whose norms are defined in accordance with the inequalities (A.1), i.e.,

$$
\|f\|_{U, b, N}=\sup _{z}|f(z)|(1+|x|)^{N} e^{-b|y|-b d(x, U)} .
$$

For each closed cone $K \subset \mathbb{R}^{n}$, one also defines a space $\mathcal{S}^{0}(K)$ by taking another inductive limit through those open cones $U$ that contain the set $K \backslash\{0\}$ and shrink to it. Clearly, $\mathcal{S}^{0}\left(\mathbb{R}^{n}\right)=\mathcal{S}^{0}$. As usual, we use a prime to denote the continuous dual of a space under consideration. A closed cone $K \subset \mathbb{R}^{n}$ is said to be a carrier of a functional $v \in \mathcal{S}^{\prime 0}$ if $v$ has a continuous extension to the space $\mathcal{S}^{0}(K)$, i.e., belongs to $\mathcal{S}^{\prime 0}(K)$. As is seen from estimate (A.1), this property may be thought of as a fast decrease - no worse than an exponential decrease of order 1 and maximum type - of $v$ in the complement of $K$. It should also be emphasized that if $v$ is a tempered distribution with support in $K$, then the restriction $\left.v\right|_{\mathcal{S}^{0}}$ is carried by $K$.

We now list some results proved by Soloviev, which formalize the property of angular localizability:

R.1 The spaces $\mathcal{S}^{0}(U)$ are Hausdorff and complete. A set $B \subset \mathcal{S}^{0}(U)$ is bounded if, and only if, it is contained in some space $\mathcal{S}^{0, b}(U)$ and is bounded in each of its norms.

R.2 The space $\mathcal{S}^{0}$ is dense in every $\mathcal{S}^{0}(U)$ and in every $\mathcal{S}^{0}(K)$.

R.3 If a functional $v \in \mathcal{S}^{\prime 0}$ is carried by each of closed cones $K_{1}$ and $K_{2}$, then it is carried by their intersection.

R.4 If $v \in \mathcal{S}^{\prime 0}\left(K_{1} \cup K_{2}\right)$, then $v=v_{1}+v_{2}$, where $v_{j} \in \mathcal{S}^{\prime 0}\left(U_{j}\right)$ and $U_{j}$ are any open cones such that $U_{j} \supset K_{j} \backslash\{0\}, j=1,2$.

\section{References}

[1] L. Álvarez-Gaumé and M.A. Vázquez-Mozo, "General properties of non-commutative field theories," Nucl.Phys. B668 (2003) 293.

[2] M. Chaichian, M.N. Mnatsakanova, K. Nishijima, A. Tureanu and Yu. S. Vernov "Towards an axiomatic formulation of noncommutative quantum field theory," hepth/0402212. 
[3] D.H.T. Franco and C.M.M. Polito, "A New Derivation of the CPT and SpinStatistics Theorems in Non-Commutative Field Theories," hep-th/0403028, revised version to appear in J.Math.Phys.

[4] W. Lücke, "PCT, spin and statistics, and all that for nonlocal Wightman fields," Commun.Math.Phys. 65 (1979) 77.

[5] W. Lücke, "Spin-statistics theorem for fields with arbitrary high energy behavior," Acta Phys.Austr. 55 (1984) 213.

[6] W. Lücke, "PCT theorem for fields with arbitrary high-energy behavior," J.Math.Phys. 27 (1985) 1901.

[7] M.A. Soloviev, "Extension of the spin-statistics theorem to nonlocal fields," JETP 67 (1998) 621.

[8] M.A. Soloviev, "A uniqueness theorem for distributions and its application to nonlocal quantum field theory," J.Math.Phys. 39 (1998) 2635.

[9] M.A. Soloviev, "PCT, spin and statistics and analytic wave front set," Theor.Math.Phys. 121 (1999) 1377.

[10] M.A. Soloviev, "Nonlocal extension of the Borchers classes of quantum fields," in Multiple Facets of Quantization and Supersymmetry, Ed. M. Olshanetsky and A. Vainshtein, contribution to Marinov Memorial Volume, World Scientific, 697-717.

[11] I.M. Gelfand and G.E. Shilov, "Generalized functions," Vol.2, Academic Press Inc., New York, 1968.

[12] H.-J. Borchers, "Über die mannigfaltigkeit der interpolierenden felder zu einer kausalen S-matrix," Nuovo Cimento 15 (1960) 784.

[13] The equivalence class of a free Hermitian scalar field was determined independently by B. Schroer (unpublished preprint) and Epstein [14]. Epstein and Schroer's theorem states that the Borchers class of the free field is made up with the set of Wick polynomials of the free field and its derivatives defined as limits of Wick polynomials, when all points are made to coincide:

$$
\lim _{x_{1}, \ldots, x_{n} \rightarrow x}: D^{\alpha_{1}} \varphi\left(x_{1}\right) \cdots D^{\alpha_{n}} \varphi\left(x_{n}\right):=: D^{\alpha_{1}} \varphi(x) \cdots D^{\alpha_{n}} \varphi(x): .
$$

In the case of a nonlocalizable field theory, the Borchers class was first raised in [15].

[14] H. Epstein, "On the Borchers class of a free field," Nuovo Cimento 27 (1963) 886.

[15] J.G. Taylor and F. Cosntantinescu, "Equivalence between non-localizable and local fields," Commun.Math.Phys. 30 (1973) 211. 
[16] N. Mahajan, "PCT theorem in field theory on non-commutative space," Phys.Lett. B569 (2003) 85.

[17] Chong-Sun Chu, K. Furuta and T. Inami "Locality, Causality and Noncommutative Geometry," hep-th/0502012.

[18] M. Chaichian, P.P. Kulish, K. Nishijima and A. Tureanu, "On a Lorentz-Ivariant Interpretation of Noncommutative Space-Time and Its Implications on Noncommutative QFT," Phys.Lett. B604 (2004) 98.

[19] V.Ya. Fainberg and M.A. Soloviev "Non-localizability and asymptotic commutativity," Theor.Math.Phys. 93 (1992) 1438.

[20] H.-J. Borchers and K. Pohlmeyer, "Eine scheinbare abschwächung der lokalitätsbedingung. II," Commun.Math.Phys. 8 (1968) 269.

[21] We recall that the Wightman functionals can be regarded as boundary values of certain holomorphic functions $[22,23,24]$, with the domain of analyticity having the form of an open tube, i.e., $T_{n-1}=\left\{\left(z_{1}, \ldots, z_{n}\right) \in \mathbb{C}^{4 n} \mid \operatorname{Re} z_{i} \in \mathbb{R}^{4 n}, \operatorname{Im} z_{i} \in V_{+}\right\}$. Here $V_{+}$is the forward light cone in $\mathbb{R}^{4 n}$, and for this reason $T_{n-1}$ is called the forward tube. $T_{n-1}$ has no real points, since the Wightman functionals are analytic only when $\operatorname{Im} z_{i} \neq 0$. An elegant technical result, the Bargamann-Hall-Wightman theorem, states the Wightman functionals can be analytically continued into the extended tube $T_{n-1}^{\text {ext }}$, called the extended forward tube, with the functionals being covariant under the complex Lorentz group $\mathscr{L}_{+}(\mathbb{C})$. $T_{n-1}^{\text {ext }}$ contains certain real points, the so called Jost points, $\mathscr{J}_{n}$, which lie outside the light cone - R. Jost, "Eine Bemerkung zum CPT," Helv.Phys. Acta 30 (1957) 409.

[22] R.F. Streater and A.S. Wightman, "PCT, spin and statistics, and all that," AddisonWesley, Redwood City, 1989.

[23] N.N. Bogoliubov, A.A. Logunov, A.I. Oksak and I.T. Todorov, "General principles of quantum field theory," Kluwer, Dordrecht, 1990.

[24] R. Haag, "Local Quantum Physics," Second Edition, Springer, Berlin, 1996. 